\title{
Study of CBNAAT and Anti-MPT64 Detection in Cytological and Histopathological Material for Early Diagnosis of TB Lymphadenitis
}

\author{
Sumayya $^{1}$, Kharidehal Durga ${ }^{2}$, B. S. Nithyananda ${ }^{3}$, Shahvar Fatima ${ }^{4}$, A. Krishnaiah ${ }^{5}$
}

\begin{abstract}
${ }^{1}$ Department of Pathology, Osmania General Hospital/Osmania Medical College, Koti, Hyderabad, Telangana, India. ${ }^{2}$ Department of Pathology, Osmania General Hospital/Osmania Medical College, Koti, Hyderabad, Telangana, India. ${ }^{3}$ Department of Pathology, Osmania General Hospital/Osmania Medical College, Koti, Hyderabad, Telangana, India. ${ }^{4}$ Department of Pathology, Osmania General Hospital/Osmania Medical College, Koti, Hyderabad, Telangana, India. ${ }_{5}^{5}$ Department of Pathology, Osmania General Hospital/Osmania Medical College, Koti, Hyderabad, Telangana, India.
\end{abstract}

\section{ABSTRACT}

\section{BACKGROUND}

Tuberculosis is primarily a pulmonary disease, but it can affect almost any organ system, with lymph node involvement being the most common form of extrapulmonary tuberculosis and tuberculous pleuritis being the $2^{\text {nd }}$ most frequent extrapulmonary manifestation. Immune-compromising diseases such as HIV, have resulted in increase in the incidence of tuberculous lymphadenitis.

\section{METHODS}

This study is a prospective study, which included 80 patients, in the age group of 1560 years, done over a period of 18 months from November 2016 to April 2018 in Upgraded Department of Pathology, Osmania General Hospital, Hyderabad, a tertiary referral centre for the state of Telangana and various districts of neighbouring states. In the present study, patients who presented with cervical lymphadenopathy as the manifestation of extrapulmonary tuberculosis were included. Cervical lymphadenopathy is the most common presentation of EPTB. Pus from the suspected lymph nodes was aspirated for cytological examination, ZN staining and GeneXpert analysis. Biopsy from the suspected lymph node was obtained and the material processed for CBNAAT, histopathology, Ziehl Neelsen staining and IHC.

\section{RESULTS}

CBNAAT was positive in $70(87.5 \%)$ cases on cytological aspirates with 24 (30\%) cases showing rifampicin resistance and on biopsy, 64/80 (80\%) cases were positive on CBNAAT with rifampicin resistance in 25 (31.25\%) cases. IHC with antiMPT64 antibody was positive in $72(90 \%)$ cases with sensitivity, specificity, positive and negative predictive value of $100 \%, 97 \%, 98 \%$ and $100 \%$ respectively and $p$ value of $<0.0001$.

\section{CONCLUSIONS}

Present study provides the insight for the feasibility of using CBNAAT and IHC as diagnostic techniques for better management of EPTB patients and be useful in settings where culture or higher molecular facilities are not available.

\section{KEY WORDS}

Extrapulmonary Tuberculosis, CBNAAT, IHC-Anti-MTB Antibody, BCG Antiserum
Corresponding Author: Dr. Kharidehal Durga, Department of Pathology, Flat No. 403, Shri Padmavathy Residency, HDFC Bank Lane, Padmaroa Nagar, Hyderabad-500025, Telangana, India. E-mail: drkdurga.path@gmail.com

DOI: $10.14260 / j e m d s / 2019 / 765$

Financial or Other Competing Interests: None.

How to Cite This Article:

Sumayya, Durga K, Nithyananda BS, et al. Study of CBNAAT and Anti-MPT64 detection in cytological and histopathological material for early diagnosis of TB lymphadenitis. J. Evolution Med. Dent. Sci. 2019;8(47):3540-3544, DOI: $10.14260 / \mathrm{jemds} / 2019 / 765$

Submission 13-08-2019,

Peer Review 07-11-2019,

Acceptance 13-11-2019,

Published 25-11-2019. 


\section{BACKGROUND}

Human Tuberculosis is a re-emerging infectious disease and has remained the world's leading cause of death from a single infectious agent. It is primarily a pulmonary disease, can affect almost any organ system, with lymph node involvement is the most common form of extrapulmonary tuberculosis and tuberculous pleuritis being the $2^{\text {nd }}$ most frequent extrapulmonary manifestation,(1) immunecompromising diseases such as HIV have resulted in increase in the incidence of tuberculous lymphadenitis.(2) Tuberculous lymphadenitis in cervical region is known as scrofula (WHO, 2015).(3) Tuberculosis is a contagious and infectious disease as M.tuberculosis, usually lasts throughout the life course and determines the formation of tubercles in different parts of the body.(4) M. tuberculosis has the ability to colonise any site in the body and it can affect organs other than lung such as pleura, lymph node, abdomen, skin, genitourinary tract, joints, bones and meninges. ${ }^{(5,6)}$ According to WHO there were 8.6 million new TB cases in 2012 and 1.3 million TB deaths, 1.4 million TB deaths in 2015, and an additional 0.4 million deaths resulting from TB disease among HIV-positive people.(7)

Extra-pulmonary tuberculosis (EPTB) accounts for 20\% (234029 cases out of 1183373) of total burden of tuberculosis globally.(7) It is estimated that approximately 70 million people will die from tuberculosis within the next 20 years and it is because of inadequate measures for the TB control.(8) Diagnosis of tuberculous lymphadenitis has been a permanent challenge, because of its severe social implications, ${ }^{(4)}$ and inability to tackle the disease due to inadequate diagnostic measures. ${ }^{(9)}$ For developing countries with large number of cases and financial constraints, identification and diagnosis of Mycobacterium tuberculosis requires rapid and inexpensive diagnostic methods and modalities.

As per WHO, the diagnosis of EPTB should be made based on relevant clinical manifestation, culture positivity, histological features of granulomatous lesion with or without caseation.(10) Granulomatous lymphadenitis can be extensive differential diagnosis for non-infective disorders like sarcoidosis, sarcoid like lymphadenitis and infective conditions like atypical mycobacterial infections, toxoplasma, syphilis, brucellosis, BCG lymphadenitis, lymphogranuloma venereum and fungal infections.(10) In countries with high prevalence, diagnosis on clinical criteria has poor sensitivity and specificity, and confirmation requires demonstration of acid-fast bacilli on $\mathrm{ZN}$ staining and/or bacteriological isolation on culture.

Most of the cases of tuberculous lymphadenitis are paucibacillary and Acid-fast bacilli has low sensitivity, as its detection limit is $>10^{4}$ bacilli/slide, or $10^{4}$ bacilli per ml(11) and Culture requires a long time of 5-8 weeks to give results, reliance on culture, the gold standard and mainstay of diagnosis often leads to delay, compromising patients care and outcomes. ${ }^{(12)}$ Diagnosis is therefore made on the classical histological changes of chronic granulomatous inflammation suggestive of tuberculosis.(13) Histology remains the most appropriate method till date, but is time-consuming to undertake and establishing a diagnosis of TB with high specificity remains difficult, as it suffers low sensitivity for paucibacillary samples.
Fine Needle Aspiration Cytology (FNAC) is less invasive, pain free OPD procedure with sensitivity and specificity of $79 \%$ and $94 \%$ respectively, ${ }^{(14)}$ and obviates the need for excisional biopsy, it is used at initial diagnosis and as well as in the follow-up post treatment of tubercular lymphadenopathy.

Nucleic acid amplification test is being increasingly used for rapid diagnosis of $\mathrm{TB}$, programmatic management and surveillance of Multi Drug Resistant- TB(MDR-TB), offering quick diagnosis, standardized testing, potential for high output and having fewer requirements for ensuring laboratory biosafety.(15) CBNAAT (GeneXpert MTB/RIF assay) was rapidly endorsed by the WHO (World Health Organization) in December 2010 as a replacement for sputum smear microscopy, particularly in settings with high rates of HIV associated TB and multidrug-resistant TB developed for testing sputum samples.(8) This multifunctional diagnostic platform, performs real-time PCR, enabling diagnosis of TB and simultaneous assessment of rifampicin resistance to be completed within 2 hours.(16) The test detects DNA specific for Mycobacterium tuberculosis by PCR.(17)

Detection of M.tuberculosis antigen by IHC is an alternative to conventional acid fast staining.(18) This study investigated the diagnostic potential of immunohistochemistry on tissue sections for specific detection of M.tuberculosis complex organisms by using an antibody to the secreted antigen MPT64, which is present only in the M.tuberculosis complex and not detected in nontuberculous mycobacteria.(19)

\section{METHODS}

Present study is a prospective study, in which 80 patients, between age group 15- 60 years were selected over a period of 18 months from November 2016 to April 2018 in Upgraded department of Pathology, Osmania general hospital, Hyderabad. Pus was aspirated from patients with suspected lymph nodes, known patients of tuberculous lymphadenitis and treatment defaulters. Excision biopsy of the lymph nodes was performed. Patients who are immunocompromised, HIV infected, malnourished, on chemotherapy, corticosteroids, or any drug except ATT were excluded from the study.

Ethical clearance was obtained from the ethics committee of the institute. Pus aspirated from the lymph node was put in a sterile container and sent for CB-NAAT and the other part was smeared on 3-4 slides. Few smears were fixed in 95\% ethyl alcohol for staining with Hematoxylin \& Eosin and the other slides were air dried for special stains e.g. ZN stain for AFB etc. On microscopic examination, the cytological findings were classified according to the amount of necrosis, the type of cells and their arrangement into necrotic granulomas, necrotic material showing mainly degenerated neutrophils, lymphocytes and epithelioid cells, and non-necrotic granulomas and AFB detected on ZN staining. For histopathological examination, lymph node biopsy material was obtained. Small part of lymph node tissue was taken and crushed into small pieces, mixed with normal saline in a sterile container and sent for CBNAAT. Remaining specimen was fixed in $10 \%$ phosphate buffer formalin overnight for conventional paraffin embedding followed by routine H\&E 
staining. Few unstained slides were prepared for Ziehl Neelsen stain by heat carbol fuchsin method.

IHC with anti-MPT64 antibody was done using peroxidase-antiperoxidase method according to the protocol described by BIOCARE. 4-mm- thick sections were taken and deparaffinized. Microwave antigen retrieval was done using citrate buffer, $\mathrm{pH} 6.2$, at $750 \mathrm{~W}$ for 10 mins and endogenous peroxidase activity was inhibited by incubating sections with hydrogen peroxide for 8 minutes. Slides were washed with TBS buffer (9.6 g of Tris Hydroxymethyl methylamine and 8.6 $\mathrm{g}$ of $\mathrm{NaCl}$ in $1000 \mathrm{ml}$ distilled water). $\mathrm{Ph}$ 7.4-7.6. Then slides were treated with primary antibodies - (i) polyclonal antiBCG, at 1/5000 dilution for 1 hour, (ii) polyclonal anti-MPT64 antibody at $1 / 250$ dilution for 1 hour. Optimal dilutions were determined priorly. The sections were washed again with TBS buffer (9.6 g of Tris Hydroxymethyl methylamine and 8.6 $\mathrm{g}$ of $\mathrm{NaCl}$ in $1000 \mathrm{ml}$ distilled water). $\mathrm{Ph}$ 7.4-7.6. Sections were incubated with anti-rabbit dextran polymer conjugated to horseradish peroxidase for 45 minutes. Antigen was visualized with 3-amino-9-ethylcarbazol- and hydrogen peroxide containing substrate and counter-stained with haematoxylin.

All incubations are carried out at room temperature and the sections are thoroughly washed in between the incubations. In every experiment, one positive control and two negative controls are included. Mycobacterial antigen staining intensity was evaluated under $40 \mathrm{X}$, total number of epithelioid cells, giant cells and the nucleated cells were evaluated for staining in each granuloma and results were categorized as weak, moderate, and strong staining subjectively.

\section{Statistical Analysis}

The data was collected and analysed using standard statistical chi - square test, $\mathrm{P}<0.05$ was taken as statistically significant. Data was entered in Microsoft excel and analysis was done using SPSS version 22.

\section{RESULTS}

In the present study, age of the patients (Table 1) ranged from 12- 65 years with mean age of 22.7 years. The maximum number of cases included were in second and third decade of life. There were 48 females and 32 males with male to female ratio of 2:3.

Cytology showed granulomatous lymphadenitis of Koch's aetiology in $73 / 80(91.25 \%)$ cases and among remaining 7 cases, $3 / 80(3.75 \%)$ cases were diagnosed as granulomatous lymphadenitis of unknown cause, and $4 / 80(5 \%)$ cases as reactive lymphadenitis, AFB by ZN staining were detected in $20 / 80(25 \%)$ cases. CBNAAT was positive in $70 / 80(87.5 \%)$ cases, with rifampicin resistant in $24(30 \%)$ cases. Statistical analysis was calculated by using chi square test, $p$ value of $<0.05$ was considered as statistically significant. CBNAAT was positive in 69 out of the 73 FNAC positive patients. In remaining 07 cases, CBNAAT was positive in 1 case, diagnosed as granulomatous lymphadenitis of unknown aetiology.
None of the ZN smear positive sample, gave negative results on CBNAAT, as well as most of the ZN smear negative samples were positive with CBNAAT, indicating Xpert MTB/RIF assay is highly sensitive and specific technique. On histopathology, both well organised and poorly organised granulomas with necrotic and non-necrotic centers were observed. Typically, well organised granulomas were observed in $72.5 \%$ cases and poorly organised granulomas and non-granulomas with only epithelioids were observed in remaining $27.5 \%$ cases. Necrosis was shown by $85 \%$ of cases.

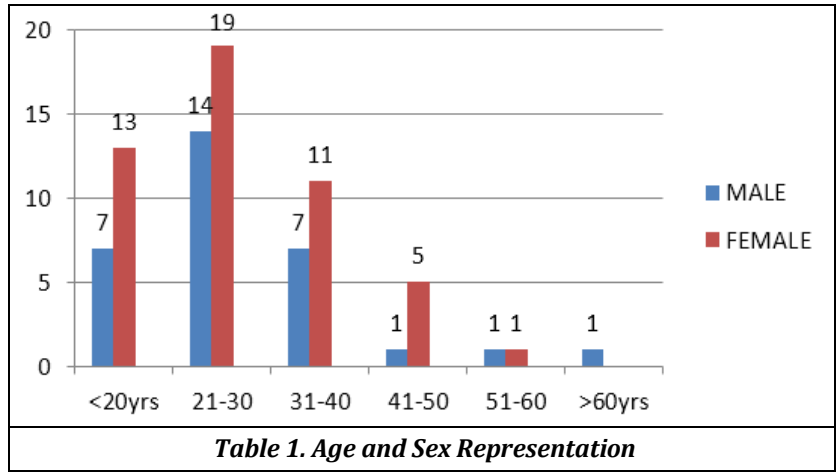

Most of the cases diagnosed cytologically, also turned out to be granulomatous tuberculous lymphadenitis on histopathology, 1 out of 3 cases, diagnosed as granulomatous lymphadenitis of unknown aetiology, 2 out of 4 cases of reactive lymphadenitis on cytology were shown to be tuberculous lymphadenitis on histology. CBNAAT was positive in $64 / 80(80 \%)$ cases with rifampicin resistance in $25(31.25 \%)$ cases. AFB were detected in 14/80 (17.5\%) cases. Initial experiments with anti-MPT64 antibody showed some cross reactivity with adjacent normal lymph node tissue and in negative controls. To enhance the specificity, the anti-MPT64 antiserum was absorbed with anti-BCG serum, which resulted in total removal of signals from adjacent normal tissue. Absorption of anti-MPT64 antiserum enhanced the specificity but did not affect the sensitivity, as there was no difference in the staining intensity in positive controls and lymphadenitis cases.

With absorbed anti-MPT64 antibody 72/80(90\%) cases were positive. MPT64 antigen was detected as granular cytoplasmic staining in the positive controls and the granuloma cells in tuberculous lymphadenitis cases. Antigen was mainly detected in epithelioid cells, giant cells and few nucleated cells. Necrotic centers were negative in the majority of cases. The intensity and extent of staining varied. Most of the cells in granulomas were strongly positive $(+2$ or $+3)$, while few cells showed moderate to weak $(+1$ or +2$)$ staining intensity. Among 72/80(90\%) cases, strong immunoreactivity was seen in $78.8 \%$ and moderate to week positivity in $21.2 \%$. 2 cases of suspected granulomatous lymphadenitis on histology, did not show any staining intensity with IHC and these turned out to be cases of foreign body granuloma and fungal granuloma confirmed by staining with Gomori methenamine silver stain. In these cases, CBNAAT and AFB were also negative. 


\section{DISCUSSION}

Extrapulmonary tuberculosis constitutes $20 \%$ of burden of TB globally.(7) EPTB being a paucibacillary disease, the number of bacteria are less to be detected and are deep seated in the organs, ${ }^{(8)}$ the diagnosis of EPTB has always been a problem. Further, conventional methods including histology, smear microscopy and AFB detection are cumbersome and never diagnostic, where culture methods are time consuming.

As per WHO, the diagnosis of EPTB should be made on the basis of culture positive specimen or caseating granuloma on biopsy or strong clinical evidence consistent with active EPTB.(10) Routine cytology and histology cannot distinguish between the diseases caused by TB or non-TB mycobacterium, or chronic inflammatory conditions. TB thus, remains undiagnosed in $20 \%$ cases even after multiple biopsies or aspirations.

GeneXpert MTB/RIF assay marks an important development in the field of rapid molecular tubercular diagnostics. This assay was rapidly endorsed by the WHO in December 2010 as a replacement for sputum smear microscopy, in the settings with high rates of HIV associated $\mathrm{TB}$ and multidrug resistant $\mathrm{TB}$, particularly for testing sputum samples.(8) Evaluations of this assay has now extended to a variety of non-respiratory clinical samples.

Extrapulmonary TB is far more complex because of diversity of clinical sample types, difficulty in obtaining adequate tissue for analysis and in the extraction of M.tuberculosis DNA from the samples.

With the improvement of nucleic acid amplification techniques in tuberculosis detection, sensitivity of test has been rising, and as this multifunctional diagnostic platform is automated and it performs real time PCR, it enables diagnosis of TB and simultaneous assessment of rifampicin resistance to be completed within 2 hours.(16) This test detects DNA specific for mycobacterium tuberculosis by PCR.(17) Genotypic methods have considerable advantages, in terms of scaling up the programmatic management and surveillance of Multi Drug Resistant-TB (MDR-TB), offering quicker diagnosis, standardized testing, the potential for high output and having fewer requirements for ensuring laboratory bio safety.(15)

Singh KG et al, showed out of total 57 cases, FNAC could diagnose tubercular cytology in 47 cases (82.4\%) and CBNAAT positive in 44 cases (77\%). In remaining 10 patients in whom FNAC was negative for TB cytology, histopathology following excisional biopsy of lymph node was positive for TB lymphadenopathy.(20) CB-NAAT done on those 10 patients before excisional biopsy on FNA sample was positive in only one patient indicating that CB-NAAT is mostly negative for MTB if cytology is also negative for TBLN. This study also shows the use and sensitivity of immunohistochemistry using anti-MPT64 antibody for the diagnosis of tuberculosis in routinely processed, FFPE histological specimens, as the expression of antigens of tubercle bacilli after in vitro culture of the organism on synthetic media are relatively well known. The strength of this technology lies in being robust, readily available in routine surgical pathology laboratories and can detect fragmented tubercle bacilli without intact cell wall.

Compared with ZN staining that has a sensitivity of 10$45 \%$ and it requires an intact cell wall, IHC offers a major improvement in diagnostic potential and should be suited for the diagnosis of pauci-bacillary EPTB. Manju R purohit et al in 2017 showed, positive immunostaining with anti-MPT64 was seen as reddish brown granular in the cytoplasm of mononuclear cells and giant cells in all TB specimens and in 1 control specimen, thus yielding a sensitivity of $100 \%$ and a specificity of $97 \%$. When a diagnostic validation of immunostaining was performed using PCR as the gold standard, the overall sensitivity, specificity, and positive and negative predictive values for immunostaining with antiMPT64 were $100 \%, 97 \%, 98 \%$, and $100 \%$, respectively.(21)

IHC for tuberculosis has, however, been slow to catch on as a routine diagnostic method in histopathology laboratories probably due to the lack of a specific anti-mycobacterial antibody suitable for all types of tissues and hence the exact diagnostic role of IHC for M. tuberculosis has to be assessed. Present study shows that IHC with anti-MPT64 has better specificity, sensitivity, and predictive values. Anti- MPT64 antibodies also gave sharp and strong signals with clear background making interpretation easier and permitting a more confident diagnosis of $\mathrm{M}$. tuberculosis complex organism.

\section{CONCLUSIONS}

Molecular diagnostic methods hold the key to the future of better and efficient diagnosis and management of EPTB. CBNAAT (GeneXpert MTB/RIF) assay is an efficient and reliable technique for rapid diagnosis of extrapulmonary $\mathrm{TB}$, with a potential to identify rifampicin resistance. Its simplicity, sensitivity, speed and automation make this technique a very attractive tool for diagnosis of mycobacterium, and can be used for programmatic management and surveillance of MDR tuberculosis. IHC with antibody to MPT64, is a specific and sensitive technique for diagnosis of EPTB, that can be used in routine laboratory. Being specific, anti-MPT-64 would be of value in differentiating M.tuberculosis from other organisms, especially non-tuberculous mycobacteria and other granulomatous inflammations. Present study provides the insight for the feasibility of using CBNAAT and IHC as diagnostic techniques for better management of EPTB patients in settings where culture or molecular facilities are not available.

\section{REFERENCES}

[1] Langer AJ, Iqbal SA, Pratt R, et al. Tuberculosis statistics in United States. Report, United States Department of Health and Human Services, Public Health Services, Centres for Disease Control, Atlanta, GA, 1989, 1991.

[2] Small PM, Schecter GF, Goodman PC, et al. Treatment of tuberculosis in patients with advanced human immunodeficiency virus infection. New England Journal of Medicine 1991;324(5):289-94.

[3] Biswas G, Das A, Haldar D, et al. et al. Clinico-pathological correlates of cervical lymphadenopathy: a hospital based study. Indian J Otolaryngol Head Neck Surg 2013;(65 Suppl 1):42-7. 
[4] Bazin H. Vaccination: a history: from Lady Montagu to Jenner and genetic engineering. Montrogue: John Libbey Eurotext 2011;30(47):6728.

[5] Cavalcante S, Chakaya JM, Egwaga SM, et al. Treatment of Tuberculosis Guidelines. $4^{\text {th }}$ edn. Geneva: World Health Organization 2010: p. 23-8.

[6] Gillespie SH. Mycobacterium tuberculosis. In: Principle and practice of clinical bacteriology. $2^{\text {nd }}$ edn. England: John Wiley \& Sons Ltd., 2006: p. 159-67.

[7] Eurosurveillance editorial team. WHO publishes global tuberculosis report 2013. Euro Surveill 2013;18(43). pii: 20615.

[8] Giri PK, Khuller GK. Is intranasal vaccination a feasible solution for tuberculosis? Expert Review of Vaccines 2008;7(9):1341-56.

[9] Lawn SD, Zumla AI. Tuberculosis. Lancet 2011;378(9785):57-72.

[10] Sumi S, Radhakrishnan VV. Evaluation of Immunohistochemistry with a panel of antibodies against recombinant mycobacterial antigens for the diagnosis of tuberculous lymphadenitis. International Journal of Medicine and Medical Sciences 2009;1(5):2159.

[11] Mfinanga SG, Morkve O, Sviland L, et al. Patient knowledge, practices and challenges to health care system in early diagnosis of mycobacterial adenitis. East African Medical Journal 2005;82(4):173-80.

[12] Avashia S, Bansal D, Ahuja K, et al. Comparison of conventional methods with gene xpert mtb/rif assay for rapid detection of mycobacterium tuberculosis and rifampicin resistance in extra-pulmonary samples. International Journal of Medical Research and Review 2016;4(2)

[13] Mfinanga SG, Morkve O, Kazwala RR, et al. Mycobacterial adenitis: role of mycobacterium bovis, non-tuberculous mycobacteria, HIV infection and risk factors in Arusha, Tanzania. East African Medical Journal 2004;81(4):1718.
[14] Ligthelm LJ, Nicol MP, Hoek KG, et al. Xpert MTB/RIF for rapid diagnosis of tuberculous lymphadenitis from fineneedle-aspiration biopsy specimens. Journal of Clinical Microbiology 2011;49(11):3967-70.

[15] Catherina CB, Pamela N, Doris H, et al. Rapid molecular detection of TB and Rifampicin resistance. N Eng J Med 2010;363:1005-15.

[16] Helb D, Jones M, Story E, et al. Rapid detection of mycobacterium tuberculosis and rifampin resistance by use of on-demand, near-patient technology. J Clin Microbiol 2010;48(1):229-37.

[17] Van Rie A, Page-Shipp L, Scott L, et al. Xpert(®) MTB/RIF for point-of-care diagnosis of TB in high-HIV burden, resource-limited countries: hype or hope? Expert Rev Mol Diagn 2010;10(7):937-46.

[18] Purohit MR, Mustafa T, Wiker HG, et al. Immunohistochemical diagnosis of abdominal and lymph node tuberculosis by detecting mycobacterium tuberculosis complex specific antigen MPT64. Diagnostic Pathology 2007;2:36.

[19] Mustafa T, Wiker HG, Mfinanga SG, et al. Immunohistochemistry using a Mycobacterium tuberculosis complex specific antibody for improved diagnosis of tuberculous lymphadenitis. Mod Pathol 2006;19(12):1606-14.

[20] Singh KG, Tandon S, Nagdeote ST, et al. A role of CBNAAT in diagnosing mycobacterial tuberculosis and rifampicin resistance in tubercular peripheral lymphadenopathy. International Journal of Medical Research and Review 2017;5(3):242-6.

[21] Purohit MR, Sviland L, Wiker H, et al. Rapid and specific diagnosis of extrapulmonary tuberculosis by immunostaining of tissues and aspirates with antiMPT64. Appl Immunohistochem Mol Morphol 2017;25(4):282-8. 